\title{
Strain distribution in single, suspended germanium nanowires studied using nanofocused $\mathrm{x}$-rays
}

Paper

Keplinger, Mario; Grifone, Raphael; Greil, Johannes; Kriegner, Dominik; Persson, Johan Mikael; Lugstein, Alois; Schülli, Tobias; Stangl, Julian

Published in:

Nanotechnology

Link to article, DOI:

$10.1088 / 0957-4484 / 27 / 5 / 055705$

Publication date:

2016

Document Version

Publisher's PDF, also known as Version of record

Link back to DTU Orbit

Citation (APA):

Keplinger, M., Grifone, R., Greil, J., Kriegner, D., Persson, J. M., Lugstein, A., Schülli, T., \& Stangl, J. (2016). Strain distribution in single, suspended germanium nanowires studied using nanofocused x-rays: Paper. Nanotechnology, 27(5), [055705]. https://doi.org/10.1088/0957-4484/27/5/055705

\section{General rights}

Copyright and moral rights for the publications made accessible in the public portal are retained by the authors and/or other copyright owners and it is a condition of accessing publications that users recognise and abide by the legal requirements associated with these rights.

- Users may download and print one copy of any publication from the public portal for the purpose of private study or research.

- You may not further distribute the material or use it for any profit-making activity or commercial gain

- You may freely distribute the URL identifying the publication in the public portal 
Strain distribution in single, suspended germanium nanowires studied using nanofocused $\mathrm{x}$ rays

This content has been downloaded from IOPscience. Please scroll down to see the full text. 2016 Nanotechnology 27055705

(http://iopscience.iop.org/0957-4484/27/5/055705)

View the table of contents for this issue, or go to the journal homepage for more

Download details:

IP Address: 192.38.67.115

This content was downloaded on 15/01/2016 at 12:01

Please note that terms and conditions apply. 


\title{
Strain distribution in single, suspended germanium nanowires studied using nanofocused $\mathrm{x}$-rays
}

\author{
Mario Keplinger ${ }^{1,9}$, Raphael Grifone ${ }^{1,2,3,9}$, Johannes Greil ${ }^{4,5}$, \\ Dominik Kriegner ${ }^{1,6}$, Johan Persson ${ }^{7,8}$, Alois Lugstein ${ }^{4}$, \\ Tobias Schülli ${ }^{2}$ and Julian Stangl ${ }^{1}$
}

\footnotetext{
${ }^{1}$ Institut für Halbleiter- und Festkörperphysik, Johannes Kepler Universität Linz, Altenbergerstraße 69, 4040 Linz, Austria

${ }^{2}$ ESRF-The European Synchrotron, 71 Avenue des Martyrs, 38000 Grenoble, France

${ }^{3}$ DESY-Photon Science, Deutsches Electronen-Synchrotron, Notkestraße 85, 22607 Hamburg, Germany

${ }^{4}$ Institute of Solid State Electronics, Vienna University of Technology, Floragasse 7, 1040 Vienna, Austria

${ }^{5}$ Department of Applied Physics, Eindhoven University of Technology, PO box 513, 5600 MB Eindhoven, Netherlands

${ }^{6}$ Faculty of Mathematics and Physics, Charles University, Ke Karlovu 5, 12116 Prague, Czech Republic

${ }^{7}$ Center for Electron Nanoscopy, Technical University of Denmark 2800 Kgs Lyngby, Denmark.

${ }^{8}$ Gemeinschaftslabor für Elektronenmikroskopie, RWTH Aachen, Ahornstrae 55, 52074 Aachen, Germany

E-mail: raphael.grifone@desy.de
}

Received 25 August 2015, revised 11 November 2015

Accepted for publication 24 November 2015

Published 12 January 2016

\begin{abstract}
Within the quest for direct band-gap group IV materials, strain engineering in germanium is one promising route. We present a study of the strain distribution in single, suspended germanium nanowires using nanofocused synchrotron radiation. Evaluating the probed Bragg reflection for different illumination positions along the nanowire length results in corresponding strain components as well as the nanowire's tilting and bending. By using these findings we determined the complete strain state with the help of finite element modelling. The resulting information provides us with the possibility of evaluating the validity of the strain investigations following from Raman scattering experiments which are based on the assumption of purely uniaxial strain.
\end{abstract}

S Online supplementary data available from stacks.iop.org/nano/27/055705/mmedia

Keywords: nanofocused XRD, germanium, single nanowire, $\mu$-Raman, strain distribution, finit element method

(Some figures may appear in colour only in the online journal)

9 These authors contributed equally.

Content from this work may be used under the terms of the Creative Commons Attribution 3.0 licence. Any further distribution of this work must maintain attribution to the author(s) and the title of the work, journal citation and DOI.

\section{Introduction}

The need for high-efficiency light emitting semiconductor devices based on silicon technology drives the efforts to alter the band structure of germanium, which has proven to be a good companion for state of the art silicon technology [1-3]. 
Its high mobility values, especially for holes, along with its compatibility with complementary metal-oxide-semiconductor processing, make it a promising material for novel on-chip light sources and detectors [4-7]. Intrinsic germanium is, however, an indirect semiconductor, with the energy minimum of the conduction band valley at the $\Gamma$ point $140 \mathrm{meV}$ higher than the global conduction band minimum at the L point [8]. Electron-hole recombination from the lowest energy valleys is therefore only possible in connection with impurity and phonon scattering, rendering this recombination inefficient as compared to direct band-gap materials. For this reason, the goal of numerous investigations is to make germanium a direct semiconductor. Various methods and theoretical predictions, providing road maps to achieve this goal, have been reported in the literature. Zone-folding in multilayer structures was pursued in the 1990's, but the resulting material combinations showed a comparatively weak transition strength [9]. Recently, theoretical improved sequences have been suggested [10], but a clear experimental proof has not been achieved so far. Direct recombination in Ge has already been shown using degenerate doping of the L valleys [11], and even lasing action has been claimed, while recent studies suggest that achieving optical gain is actually more complex than originally assumed [12]. Alloying Ge with Sn was reported to result in direct band-gap behaviour [13], and even lasing has been demonstrated recently [14] despite difficult material control due to the low solubility of $\mathrm{Sn}$ in Ge.

Another promising attempt is strain engineering, which turns out to be a powerful tool for altering the band structure of semiconductor materials [15]. By applying uniaxial tensile strain along the $\langle 111\rangle$-direction in the order of $4 \%$ on single crystal Ge, quasi-particle calculations within the GW approximation predict a direct band gap at the $\Gamma$ point [16]. This would give the possibility of highly efficient optical emission, however, the required strain values are difficult to realize experimentally. Efforts have been made to realize strained $\mathrm{Ge}$ layers on top of various substrates. Here lattice mismatch and divergent thermal expansion coefficients, together with strainenhancing microstructuring, were used to apply strain to the $\mathrm{Ge}$ layer [17]. Enhanced photoluminescence emission was obtained, but eventually direct band-gap Ge based on strain engineering alone has not yet been reached. Nanowires (NWs) have proven to bear very high tensile strain values, of up to $10 \%$ strain before mechanical fracture has been reported in certain cases [18]. This makes them interesting candidates for achieving uniaxially strained Ge with sufficiently high strain values, just by stretching them in the axial direction. However, it is as important as it is nontrivial to fabricate devices which provide elongated NWs. Sample layouts based on lithographically structured SOI substrates have been developed for this purpose [19], resulting in devices with single prestrained germanium NWs. Actually, most of the concepts mentioned above include strained material, eventually in combination with other approaches, and very often micro- or nanostructuring is an important step in device design. Thus local strain determination is very important, and confocal-focused $(\mu)$-Raman scattering is often used to locally measure the strain state [20, 21], usually using bulk strain shift coefficients and assuming a particular, e.g., uniaxial, strain state. Moving towards nanostructures, confinement effects and surface states might lead to alterations of the strain shift coefficients involved in the analysis, and in real devices deviations from a purely uniaxial strain state might occur. This calls for a more direct and independent method to analyse the NW's strain state.

We present nanofocused $\mathrm{x}$-ray diffraction (XRD) experiments and perform complementary finite element method (FEM) [22] modelling to provide a detailed insight into the strain state of tensile strained suspended Ge NWs. Those findings are then compared with $\mu$-Raman experiments on the very same nanostructures. Due to the particular device design [19], besides uniaxial tension, the NWs also exhibit a certain bending, i.e. curvature, resulting in a more complex strain distribution within the nanowires. Moreover, the NW geometry, in particular a pronounced tapering and the presence of an amorphous shell around the bottom part of the wires, additionally affect the resulting strain distribution. Since it is mandatory to perform the diffraction experiment only on single, selected nanowires, we use nanofocused XRD: a full width half maxima (FWHM) focal-spot size of around $400 \mathrm{~nm}$ allows for the illumination of parts of the NWs and for following the strain distribution along their growth direction. FEM modelling also gives access to details in the strain distribution and shear strains, which are not directly measured and are deduced from the measured NW bending and geometry. Furthermore, the Raman shifts were calculated using the strain tensor from FEM simulations for a direct comparison of the values obtained by $\mathrm{XRD}$ and the measured Raman shift for several positions along the nanowires.

\section{Sample structure}

The basis for the straining devices have been silicon on insulator (SOI) wafers consisting of a bottom Si (111) handle wafer and a highly boron doped Si (110) device layer separated by silicon dioxide, as shown in figures 1(a) and (b). Using photolithography, reactive ion etching, and partial removal of the buried oxide by wet etching, a mesa structure with freestanding Si cantilevers with $\{111\}$ faceted sidewalls was fabricated according to [19]. Moreover, mesa patches with side walls parallel to the cantilever's sidewall were located opposite the cantilevers, providing mesa-cantilever gaps which were eventually bridged by Ge NWs. The cantilevers were prestrained prior to $\mathrm{NW}$ growth, and later released, so that finally the NWs bridging the gap between mesa and cantilever take up this strain. For more details please refer to the supporting information.

Top view scanning electron microscopy (SEM) images, see figures 1(c) and (d), show the final two devices investigated by nanofocused XRD and $\mu$-Raman scattering. The NWs have similar lengths of around $3 \mu \mathrm{m}$, and the cantilevers are $1.9 \mu \mathrm{m}$ thick in both devices. Apparently, the NWs exhibit 

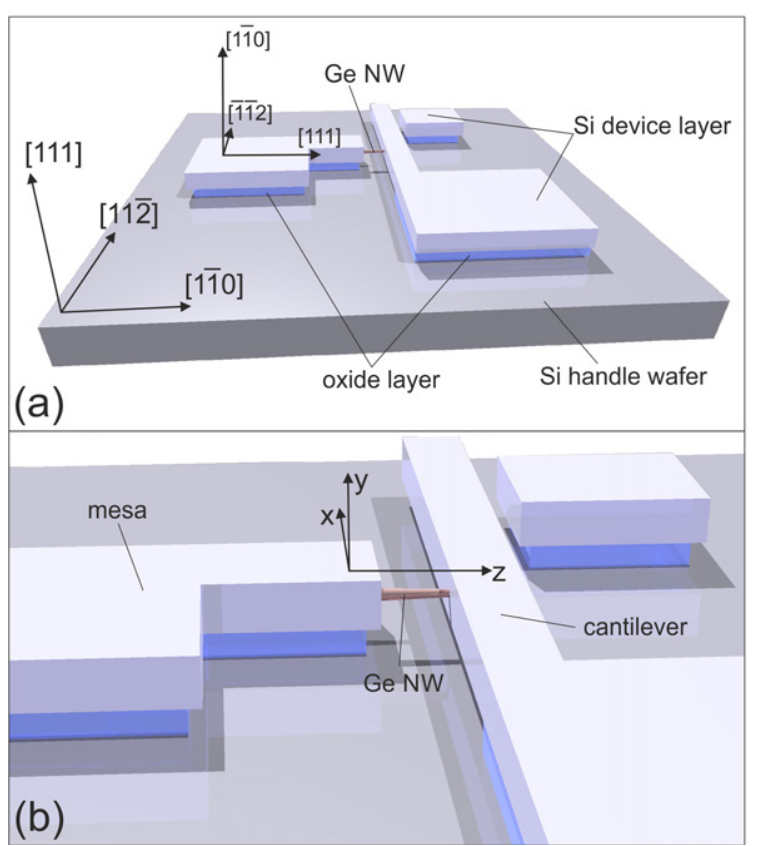

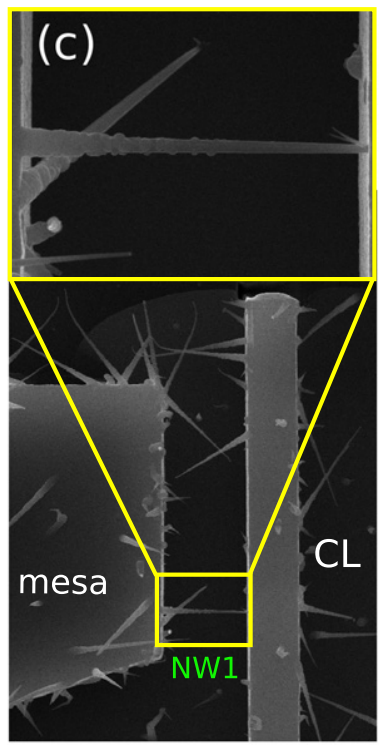

NW1: $3.2 \mu \mathrm{m}$

CL: $1.9 \mu \mathrm{m}$

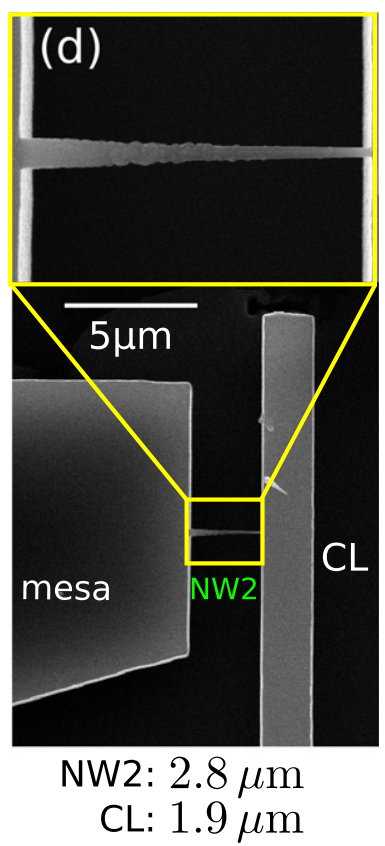

CL: $1.9 \mu \mathrm{m}$

Figure 1. (a) Sample sketch overview and close-up (b). The sample is fabricated from an SOI wafer with a [111]-oriented handle wafer and a [110]-oriented device layer. The Ge NW (red) grows between the left mesa and the cantilever, which both have [111]-oriented side facets. An additional mesa (top right of sample) serves to prebend the cantilever, see supplementary material for details. The close-up in (b) shows the orientation of the (xyz) coordinate system used below. (c)-(d) Top view SEM images of the investigated devices. Shown are the freestanding cantilever (CL) and opposite it, a mesa pad, which together create the gap where the suspended and strained NW is located. The NW lengths as well as the cantilever width are specified at the bottom for NW1 and NW2, respectively.

a pronounced tapering in growth direction. Apart from the crystalline Ge core the NWs are covered by a buckled, tapered shell, as seen in the insets at the top of figures 1(c) and (d). Transmission electron microscopy images taken from the sample show that this shell is amorphous around a crystalline Ge core ${ }^{10}$. Hence one cannot expect an x-ray diffraction signal from this shell. We will come back to this in more detail during the discussion of the results, in section 5 .

\section{Experiments}

Confocal $\mu$-Raman scattering experiments have been carried out following the scheme detailed in [20]. The excitation laser was focussed to a diffraction limited spot of $\approx 500 \mathrm{~nm}$ and centred in the gap on the suspended NW. Raman spectra were recorded in backscattering geometry. The linearly polarized laser, with power output $\approx 40 \mu \mathrm{W}$, was sent through a dichroic beam splitter onto the sample. The emitted light was then filtered by a Shamrock $532 \mathrm{~nm}$ RazorEdge long-pass edge filter, being coupled into a single-mode fibre waveguide from the microscope into the spectrograph with an $\mathrm{f} / 4$ aperture ratio. Gratings with $600 \frac{\mathrm{l}}{\mathrm{mm}}$ and $1800 \frac{\mathrm{l}}{\mathrm{mm}}$ and a $300 \mathrm{~mm}$ focal length were used in the spectrograph. Raman measurements were performed in the centre of the investigated

10 Such images are reported in [20] and can be found in the supporting information of this work, available at stacks.iop.org/nano/27/055705/ mmedia.
NWs as well as in two spots close to the base (bottom) and the top end (tip) of the NWs.

The nanofocused XRD experiments were performed at beamline ID01 of the European Synchrotron (ESRF) in Grenoble, France. A Fresnel zone plate was used to focus a monochromatic incident x-ray beam to a FWHM spot size of $\approx(400 \times 400) \mathrm{nm}^{2}$ at an energy of $8.9 \mathrm{keV}$. The experiment and data analysis will be elaborated for NW1; NW2 is analysed in the same manner and any particular differences will be mentioned explicitly. Due to the fact that the surface orientation of the SOI wafer's device layer is a [110] direction, the NW's [1 110$]$ orientation is also parallel to the sample surface normal for the selected NWs, which grow along the mesa sidewall's surface normal, i.e. the [111] direction. Due to the particular geometry, only the [22̄0] Bragg reflections have been available at the wavelength used, which means that we could directly measure the strain $\epsilon_{\perp}$, in the direction perpendicular to the NW growth direction. The scattered intensity from the Ge $(2 \overline{2} 0)$ Bragg reflection was collected with the scattering plane aligned perpendicular to the wire, i.e., along the gap between mesa and cantilever (see figure 2(a)). In order to bring the nanowire exactly into the focal spot, the sample was pre-aligned with an optical microscope mounted on the diffractometer and looking from the top onto the sample. The optical microscope was aligned to the centre of rotation of the goniometer, which coincides with the X-ray's focal spot. Given the limited precision of the bearings of the goniometer stages, this alignment is accurate up to few micrometers. The finer position determination was then done with scanning $\mathrm{x}$-ray diffraction (SXRD) 


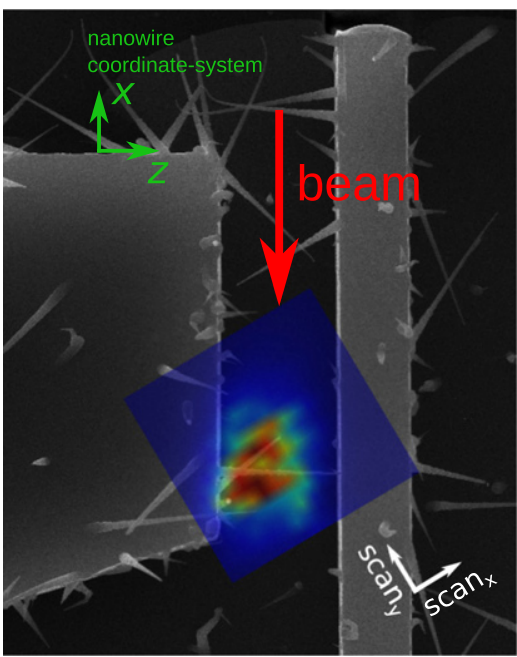

(a)

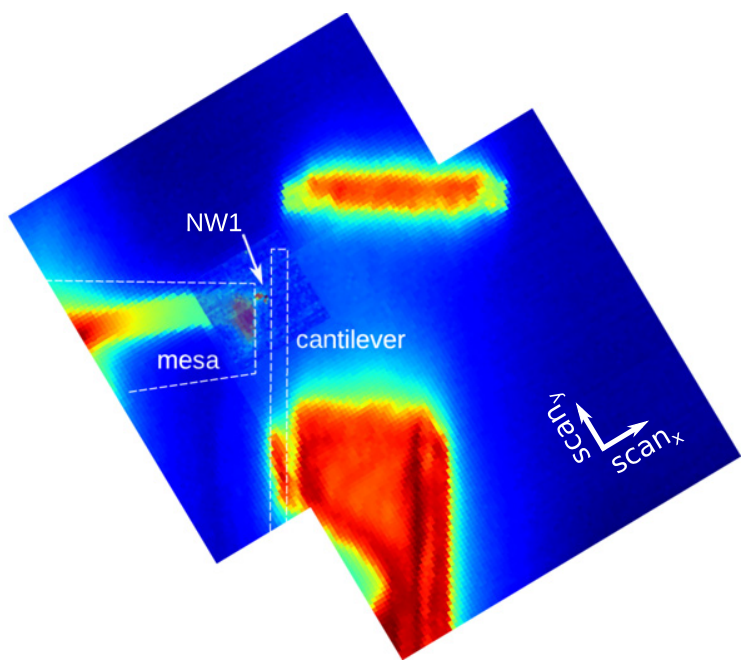

(b)

Figure 2. (a) Overlay of the Ge (22̄0) SXRD map and an SEM image of NW1. The direction of the incoming nanofocused $x$-ray beam is indicated with a red arrow. The coordinate systems of the piezoelectric scanner scan $x, y$ and of the mesa/NW crystal lattice $x, y, z$ are indicated. (b) Resulting real-space map of SXRD microscopy at the Si and Ge (22̄0) Bragg reflections. The cantilever and parts of the mesa (white dashed lines) are not visible, i.e. not in Bragg condition due to its torsion and bending.

microscopy [23]: moving the goniometer angles first to the $\mathrm{Si}$ $(2 \overline{2} 0)$ Bragg condition, the scattered intensity is recorded as a function of the sample's lateral position, which is controlled by a piezo stage moving with nanometer precision [24]. This allows us to detect the exact position of the mesa and the cantilever. Then, the Bragg angles are changed to the Ge $(2 \overline{2} 0)$ Bragg condition and the Ge nanowire position is determined in the same fashion. The resulting real-space maps are shown in figure 2(b).

Three-dimensional reciprocal-space maps (RSMs) around the Ge (220) Bragg reflection were recorded for each real-space position of the focal spot with respect to the investigated NW. This was achieved by measuring the scattered intensity using a two-dimensional pixel detector (MAXIPIX) for a series of incidence angles around the probed Bragg angle. Since the piezo-driven spatial motion is much faster than the goniometer rotations, a real-space map was recorded for each incidence angle of the primary beam, which was changed in $0.1^{\circ}$ steps, roughly corresponding to the divergence of the focused incident beam. The real-space grid axes are thereby defined by the real-space directions $\operatorname{scan}_{x}$ and $\operatorname{scan}_{y}$. The focal spot size of the focused beam defined the step size of the real-space grid in $\operatorname{scan}_{x, y}$ directions (note that these axes are rotated by $45^{\circ}$ with respect to the crystalline coordinate system $\{x, y, z\}$, as indicated in figure 2). An inset in figure 2(a) shows the scattered intensity from the Ge NW, integrated over the whole Bragg reflection and overlayed to an SEM image of the particular NW. The wire appears significantly broadened due to the convolution of its actual size with the focused synchrotron beam diameter, which is additionally projected to the surface by the Bragg angle of $\approx 17^{\circ}$.

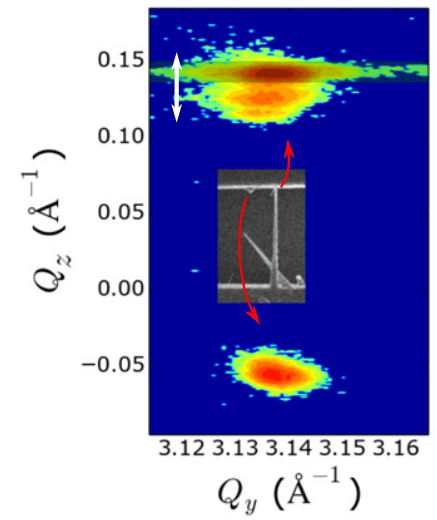

(a)

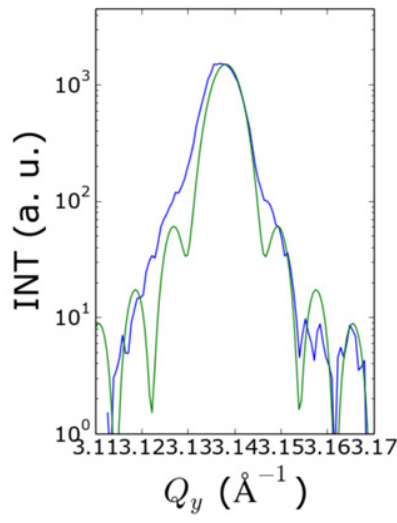

(b)
Figure 3. RSM of NW1; recorded while the focused beam was positioned at the NW tip. On the left hand side a $Q_{y}-Q_{z}$ slice is shown where the intensity distribution from the NW is located at positive $Q_{z}$ values and a second signal is located at negative $Q_{z}$ values. The origin of this second reflection is a crystallite on the cantilever's side wall. The inset of a SEM micrograph along with red arrows illustrate this situation. On the right hand side the intensity distribution from the NW in the $Q_{y}$ direction, integrated over an area illustrated by a watermark in the left plot, is shown (blue line). This is compared with the shape function of a hexagon with an $80 \mathrm{~nm}$ diameter (green line). White arrows in (a) indicate the elongation of the NW reflection in the $Q_{z}$ direction due to bending (see text for details).

\section{XRD data evaluation}

An example of the recorded RSMs of NW1, containing all interesting features for our discussion, is shown in figure 3(a). On top of the $Q_{y}-Q_{z}$ slice, i.e. for positive $Q_{z}$ values, one can 
see the scattered intensity from the NW tip $\left\{Q_{x}, Q_{y}, Q_{z}\right\}$ with thickness oscillations in the $Q_{y}$ direction ${ }^{11}$. This finding confirms the SEM images, which showed that the side facets of the hexagonal NW are parallel to the sample surface. The observed intensity oscillations were then compared to simulations, i.e., the Fourier transform of a hexagonal shape shown in figure 3(b), yielding tip diameters of $80 \mathrm{~nm}$ and $64 \mathrm{~nm}$ for NW1 and NW2, respectively. From the position of the NW's Bragg reflection the lattice plane spacing in the [110] direction is determined as detailed below. The NW signal is additionally broadened in the $Q_{z}$ direction (along the NW growth direction, indicated by the white arrow). This reflects bent lattice planes within the illuminated NW segment $^{12}$. Additionally, a second diffraction signal was recorded at negative $Q_{z}$ values. This signal actually stems from a $\mathrm{Ge}$ crystallite located on the side facet of the cantilever, as illustrated in the inset of figure 3(a). The crystallite is expected to be grown epitaxially on the cantilever, except for a very small possible tilt due to a few misfit dislocations. Hence, the orientation of this Bragg reflection was taken as a reference direction in reciprocal space, giving the surface normal of the cantilever's side facet. This represents a local crystal direction very close to the actual measured NW position on the cantilever. By comparing this reference with the NW Bragg reflection's position one is able to calculate the NW's bending and tilting with respect to the cantilever. Here, 'tilt' denotes the angle between the cantilever's surface normal and the NW growth direction, whereas 'bending' denotes the NW's curvature. In order to analyse both the lattice parameter and bending quantitatively we have to reduce the NW signal which was acquired as a 5D data set (three reciprocal space and two real space coordinates) to a $2 \mathrm{D}$ data set depending only on the real-space positions. For this the following formalism was applied:

The reciprocal-space positions $Q_{x}, Q_{y}$, and $Q_{z}$ along with the respective intensity values $I_{\text {grid }}^{220}(\vec{Q})$ were transformed according to

$$
\mathrm{F}_{\text {grid }}^{2 \overline{2} 0}=\frac{\sum_{Q_{x} Q_{y} Q_{z}} I_{\text {grid }}^{2 \overline{0} 0}(\vec{Q}) f(\vec{Q})}{\sum_{Q_{x} Q_{y} Q_{z}} I_{\text {grid }}^{2 \overline{2} 0}(\vec{Q})}
$$

and

$$
\mathrm{F}_{\text {grid }}^{2 \overline{2} 0}=\sqrt{\frac{\sum_{Q_{x} Q_{y} Q_{z}} I_{\text {grid }}^{2 \overline{2} 0}(\vec{Q}) f(\vec{Q})^{2}}{\sum_{Q_{x} Q_{y} Q_{z}} I_{\text {grid }}^{22 \overline{2} 0}(\vec{Q})}-\mathrm{F}_{\text {grid }}^{2 \overline{2} 0^{2}}}
$$

The label 'grid' denotes the variable's dependency on $\operatorname{scan}_{x}$ and $\operatorname{scan}_{y}$. Since it is important to evaluate the reciprocal-space signal only when the focused $\mathrm{x}$-ray beam was actually illuminating the NW, we disregarded data below a certain intensity threshold. In order to deduce the lattice plane spacing

11 The reciprocal coordinates $\left\{Q_{x}, Q_{y}, Q_{z}\right\}$ are parallel to the nanowire coordinate system $\{x, y, z\}$ apart from a small tilt of a few degrees of the NW.

12 This Bragg peak elongation in the $Q_{z}$ direction is actually the convolution of the bending distribution with the divergence of the nanofocused beam.
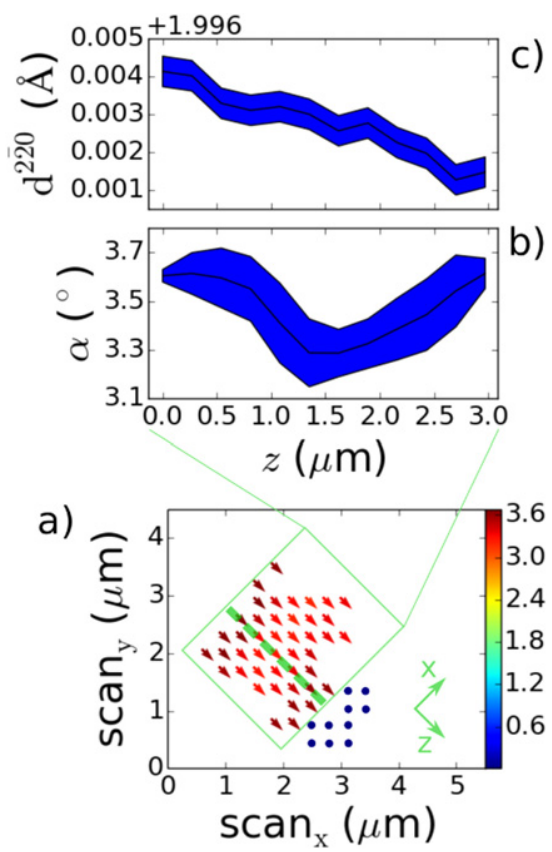

Figure 4. (a) Tilt and bending map of NW1, calculated from all Bragg peak positions with respect to the crystallite's Bragg peak (blue dots with $0^{\circ}$ ), at different real-space positions. The arrows indicate the direction, while the bending values are colour coded in degrees. For illustration reasons the green dashed line indicates the position of the NW. Line plots of the measured bending distribution $\alpha$ and the lattice plane spacing $\mathrm{d}^{(2 \overline{2} 0)}$ along the $\mathrm{NW}$ growth axis are shown in (b) and (c), respectively. The blue area represents the measurement error in graph (c). However, in graph (b) the blue area reflects the lattice bending and the divergence of the nanofocused beam.

and thus the strain in this direction, i.e. $\epsilon_{\perp}$, the function $f(\vec{Q})=\frac{2 \pi}{\sqrt{Q_{x}^{2}+Q_{y}^{2}+Q_{z}^{2}}}$ is used in equations (1) and (2). From this, one obtains the lattice plane spacing $\mathrm{d}_{\text {grid }}^{2 \overline{2} 0}=\mathrm{F}_{\text {grid }}^{2 \overline{2} 0}$ and its standard deviation $\sigma_{\mathrm{d}_{\text {grid }}^{220}}=\sigma_{\mathrm{F}_{\text {grid }}^{220}}$. The deduced lattice plane spacings represent averages within every illuminated NW segment and the values of $\sigma_{\mathrm{d}_{\text {grid }}^{220}}$ reflect the width of the NW Bragg reflection in the $Q_{y}$ direction, which is governed by the divergence of the nanofocused beam $\left(\approx 0.1^{\circ}\right)$. Its tilt can be obtained using again equations (1) and (2) by introducing $f(\vec{Q})=\arccos \left(\frac{\vec{Q}_{\text {ref }} \cdot \vec{Q}}{\left|\vec{Q}_{\text {ref }}\right||\vec{Q}|}\right)$, resulting in the values $\alpha_{\left(\operatorname{scan}_{x}, \operatorname{scan}_{y}\right)} \equiv \alpha_{\text {grid }}$. For NW1, $\vec{Q}_{\text {ref }}$ was the position of the crystallite's Bragg reflection, as mentioned. The tilt distribution measured via $\sigma_{\alpha_{\text {grid }}}$ gives the experimental value for the bending of the NW in each segment. Both tilt and bending are shown in figure 4(a) as a function of $\operatorname{scan}_{x}$ and $\operatorname{scan}_{y}$, with tilting directions indicated as arrows and the magnitude colour coded in degrees. The position of the NW is represented by a light green dashed line. As for the $\mathrm{d}_{\text {grid }}^{2 \overline{2} 0}$ values, also $\alpha_{\text {grid }}$ is practically constant perpendicular to the investigated NW, since the 'smearing' of the diffracted signal along this real space direction is only due to the distribution of a specimen/ beam-geometric configuration. To obtain the lattice plane spacing within the illuminated NW segments along the NW, the values of $\mathrm{d}_{\text {grid }}^{2 \overline{2} 0}$ were averaged in the direction perpendicular 
to the NW's growth axis with $\sigma_{\mathrm{d}_{\text {grid }}^{2 \text { rid }}}$ used as a weighting function. Since the width of the NW signal in real space is due to a geometrical broadening effect as discussed above, the values are practically constant perpendicular to the NW growth direction, and the integration mainly reduces the noise in the curve. To get the bending/tilt variation $\alpha$ along the investigated NW, the grand means of $\alpha_{\text {grid }}$ were calculated in the direction perpendicular to the NW's growth axis, and the result is shown in figure 4(b). Moreover, the according standard deviations $\sigma_{\alpha}$ were calculated for each illuminated NW segment. A tilt of $3.6^{\circ}$ with an additional bending of around $0.4^{\circ}$ was found for NW1. High-resolution SEM images, not shown here, recorded under a sample tilt of $30^{\circ}$, proved that the NW was indeed not growing parallel to the surface but under a certain angle. Figure 4(c) shows the resulting values of $\mathrm{d}^{2 \overline{2} 0}$ and $\sigma_{\mathrm{d}^{220}}$ along NW1. Comparing those values of $\mathrm{d}^{2 \overline{2} 0}$ with the bulk germanium lattice plane spacing $\frac{\mathrm{a}_{\mathrm{Ge}}}{\sqrt{h^{2}+k^{2}+l^{2}}}$ according to the (220) Bragg reflection and $\mathrm{a}_{\mathrm{Ge}}=5.658 \AA$ [25] finally yields the radial strain, i.e. $\epsilon_{\perp}$, of the distorted NW.

For NW2, no particular reference direction in reciprocal space was available, because the $\mathrm{Si}$ signal (from mesa or cantilever) was too far away and significant goniometer movements were necessary to bring it onto the detector. Hence, the bending of this NW was calculated with the NW tip's Bragg reflection position as a reference.

\section{Results and discussion}

In order to also analyse the strain $\epsilon_{\|}$along the NW growth axis from the measured strain $\epsilon_{\perp}$ and tilt/bending values, FEM simulations were performed. The orientation of the strain tensor used for the simulations with respect to the lab system was defined as follows: the normal axis $\epsilon_{y y}$ and $\epsilon_{z z}$ were chosen parallel to $\epsilon_{\perp}$ and $\epsilon_{\|}$respectively. The material constants from [25] for the crystalline Ge NW-core itself, and from [26] for the amorphous germanium/oxide shell. Since the wires have been kept in air for a long period of time during precharacterization and transport to the synchrotron, the shell is most likely oxidized. The relaxation of the prebent cantilever can, beside the desired NW tension, cause additional forces on the NW. For example, a small vertical displacement of the cantilever would apply a force on the NW tip perpendicular to its growth direction, hence resulting in a bending of the NW. Another possible load case would be if the cantilever was to become slightly twisted, which can also result in a certain NW bending. Due to the tapering and inhomogeneous shell, this finally leads to an inhomogeneous strain distribution along and within the wires. The geometric dimensions from high-resolution SEM and XRD (analysis of finite thickness fringes) have been used as input for the FEM model: the NW tapering and the amorphous Ge shell at the thicker end (which is also tapered) have been taken into account. Special boundary conditions account for the influence of mesa and/or cantilever structures straining the NW. The bottom face of the NW was fixed, reflecting the rigid
$\mathrm{NW} /$ mesa connection at the initial growth point. At the thin tip end of the NW two forces, $F_{1}$ and $F_{2}$, were applied; a tensile force $F_{1}$ which elongates the NW due to the relaxing cantilever after FIB cutting, as well as a force $F_{2}$ due to the vertical positioning of the cantilever. This second force has direct effects on the bending of the NW. The exact origin and magnitude of these vertical (along the crystallographic $y$-axis) cantilever movements are not investigated here; they may be due to inhomogeneous oxidation conditions or to the influence of the NW itself, connecting mesa and cantilever not necessarily in a symmetric fashion. Due to the cantilever tilting, not only an up/downward deflection of the NW tip can occur, but also a rotation of the NW tip facet with respect to the bottom facet, which is also included as a parameter in the FEM simulations. Since we have no means of directly assessing the forces, we use them as fit parameters in the simulations. Calculations were run to obtain the best match to the measured parameters, which are the NW strain in the [110] direction and the bending values along the wire length. To account for the sampling of NW segments with the nanofocused X-ray beam, the simulated NWs were divided into segments according to the selected $\operatorname{scan}_{x}$ and $\operatorname{scan}_{y}$ step widths. Just the simulated strain values from the crystalline core were evaluated, which reflects the fact that the measurement is sensitive solely to the crystalline parts of the NW. The resulting strain values, as well as the bending angles, were then averaged within these segments. Moreover, the standard deviation of the distributed values was calculated to give a measure of how much the values are scattered within one segment.

The concluding simulation results for NW1 and NW2 are found in table 1. Illustrations of the simulated NWs are found in figures 5 and 6.

The results for NW1 are presented in figure 5, where the coordinate system of the simulation is depicted in the inset at the top along with an on-scale sketch of the simulated geometry at the bottom. The measured strain values of $\epsilon_{\perp}$, shown as a black error bar plot in panel (a), vary along the NW due to its tapering as well as the amorphous shell, reaching a maximum strain of $-0.14 \%$ at the NW tip. For the measured values, the error bars reflect the standard measurement error. The blue line, together with the dark and light blue areas, show the according simulation results for the optimum parameters from the fit procedure. The shaded areas reflect the minimum and maximum values in the simulated segments (light blue) and the standard deviation of the strain distribution (dark blue). The measured values of $\alpha$ depending on the position along the NW are plotted in figure 5(b). Here the error bars of the measured values are given by the elongation of the NW reflection, see figure 3 , and are thus an actual measured bending distribution, i.e. a curvature, within the illuminated NW segment.

A force $\left(F_{1}\right)$ of $8.60 \mu \mathrm{N}$ in the direction of the NW tip front face's surface normal was applied to stretch the NW by approximately $18 \mathrm{~nm}$. However, applying only this force would decrease the NW tilt angle and the bending would be incompatible with the experimental data. Thus a force $F_{2}$ with a strength of $0.536 \mu \mathrm{N}$, which accounts for the second effect 
Table 1. List of all simulation parameters resulting in the best agreement between the measured strain values and the simulation results. The NW dimensions were specified from SEM micrographs and the NW tip diameters have additionally been confirmed by the evaluation of thickness oscillations in the recorded XRD spectra. All lengths are given in $\mathrm{nm}$.

\begin{tabular}{lccccccccc}
\hline & \multicolumn{4}{c}{ Core } & & \multicolumn{2}{c}{ Shell } & & \\
\cline { 2 - 4 } & Tip $\phi$ & Bottom $\phi$ & Length & & Bottom $\phi$ & Length & $F_{1}(\mu \mathrm{N})$ & $F_{2}(\mu \mathrm{N})$ \\
\hline NW1 $^{\text {a }}$ & 80 & 125 & 3200 & & 290 & 900 & 8.60 & 0.536 \\
NW2 & 64 & 120 & 2800 & & 300 & 2000 & 6.30 & 0.007 \\
\hline
\end{tabular}

${ }^{a}$ To mimic the NW tilt, an inclination of $3.6^{\circ}$ was applied to both NW end surfaces.
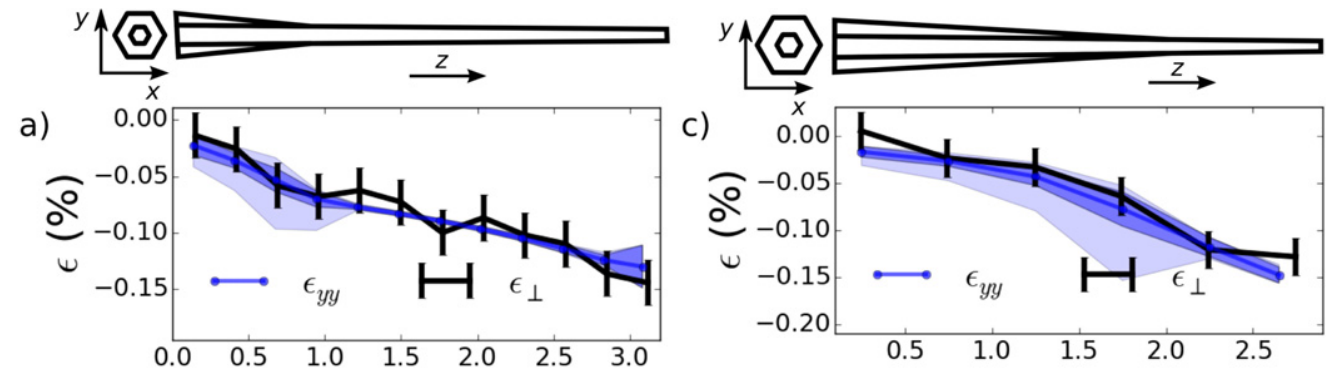

b)

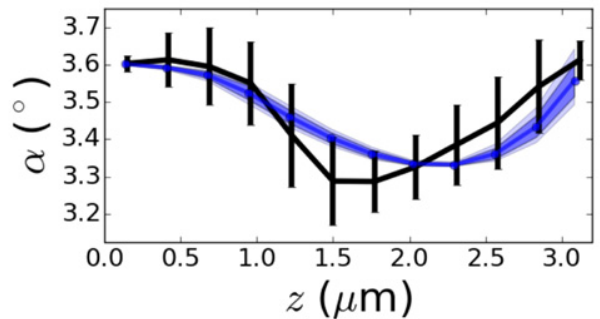

d)

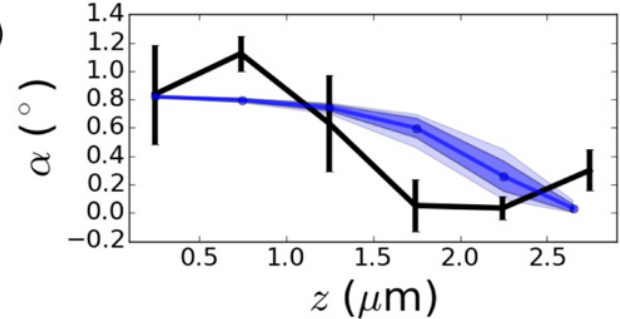

Figure 5. Results for NW1 and NW2, respectively. In (a) and (b) the measured strain in the [110] direction $\left(\epsilon_{\perp}\right)$ is shown in black, and in (c) and (d) the bending/tilting along the NW is also shown in black. The blue lines along with the dark and light blue areas illustrate the according FEM simulation results. The lines show the average values in each NW segment, the light blue areas indicate the minimum and maximum values and the dark blue areas show the standard deviation of the distribution of strain and bending/tilting values. The according simulation parameters are found in table 1 , while the sketch at the top illustrates the wire geometry used, including a tapered oxide shell starting at $z=0$.
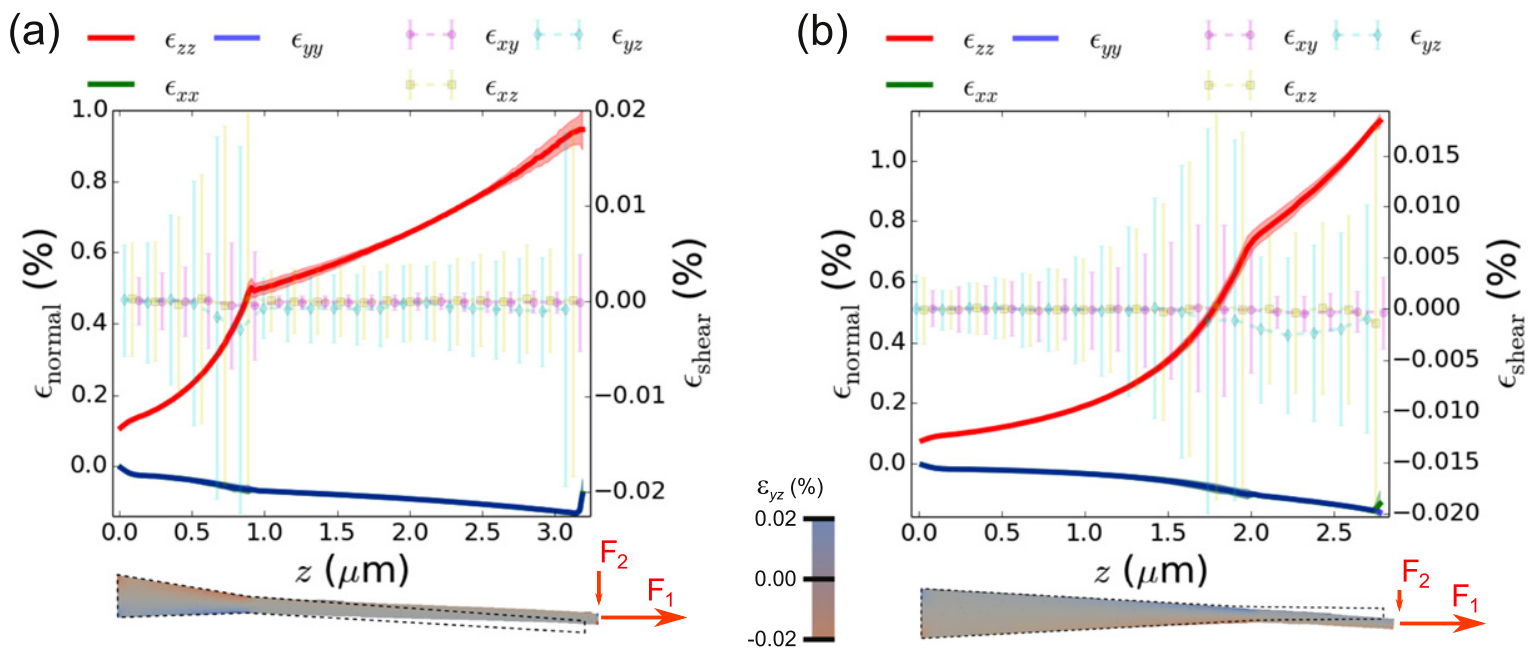

Figure 6. Strain discussion for NW1 (a) and NW2 (b). The strain tensor values within the simulated crystalline core wire, averaged in the NW's radial direction, are plotted along the NW's axial direction. Due to the existence of strain distributions in the radial direction the standard deviation of this distribution is shown additionally as light areas for the symmetric strain components and as error bars for the asymmetric strain components. To take the huge magnitude difference of the symmetric and asymmetric components into account, they are illustrated within two different $y$-axes. Actual slice plots from the FEM simulations illustrate the geometric configuration of the NWs and the applied forces. This two-dimensional colour plot shows the shear strain component $\epsilon_{y z}$ and for a proper illustration of the NW deformation it was enhanced by a factor of five. 

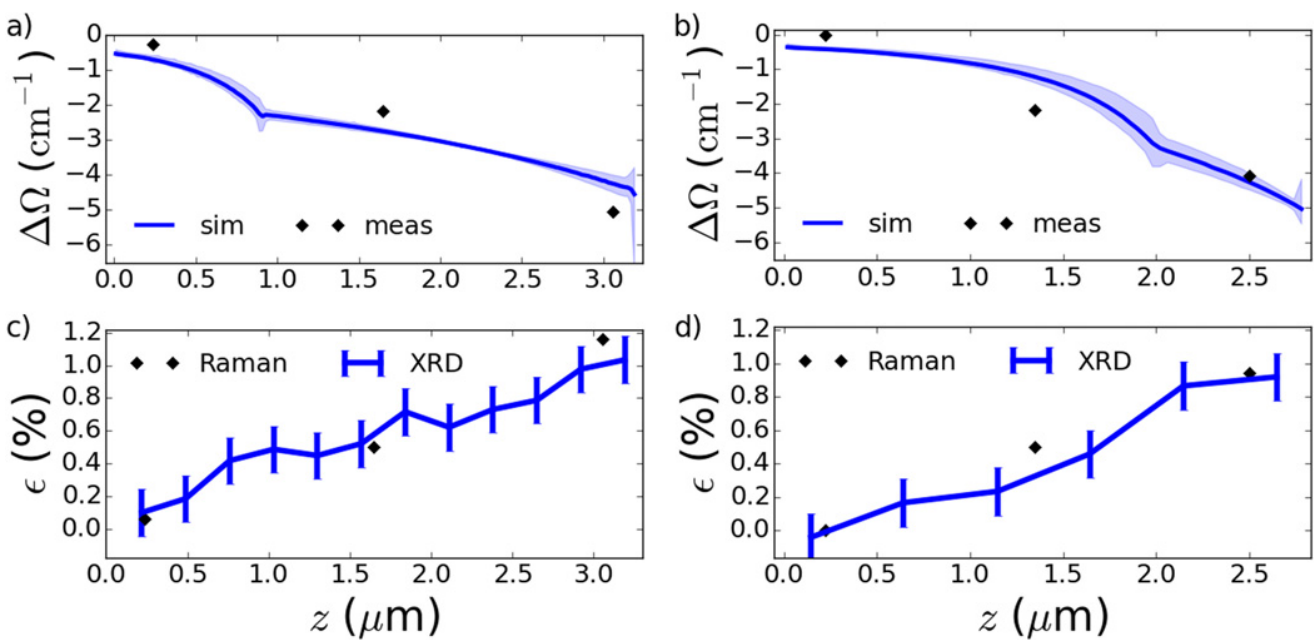

Figure 7. Comparison of the Raman simulation (blue) and the measured Raman shift (black diamonds) for NW1 (a) and NW2 (b). The blue areas show the minimum and maximum strain values from the radial strain variation within the NWs. (c) and (d) show the corresponding strain $\epsilon_{\|}$for the assumption of an uniaxial applied strain and a bulk Poisson ratio, calculated from Raman data (black diamonds) together with the XRD data (blue).

Table 2. The resulting Raman shift from the $\mu$-Raman scattering experiment along with the according strain values calculated with the conversion factor $k=434 \frac{1}{\mathrm{~cm}}$. As the reference position of the unstrained germanium first order optical phonon peak, $\omega_{0}=300.5 \frac{1}{\mathrm{~cm}}$ is used. These values are compared to XRD results, derived from the Poisson's ratio for bulk Ge.

\begin{tabular}{lccccccc}
\hline & \multicolumn{3}{c}{ NW1 } & & \multicolumn{3}{c}{ NW2 } \\
\cline { 2 - 4 } \cline { 7 - 8 } & Bottom & Middle & Tip & & Bottom & Middle & Tip \\
\hline$\Delta \Omega\left(\mathrm{cm}^{-1}\right)$ & 0.27 & 2.18 & 5.05 & & 0.0 & 2.16 & 4.09 \\
$\epsilon_{\|}(\%)($ Raman $)$ & 0.06 & 0.5 & 1.16 & & 0.0 & 0.5 & 0.94 \\
$\epsilon_{\|}(\%)($ XRD $)$ & 0.1 & 0.58 & 1.03 & & -0.04 & 0.35 & 0.92 \\
\hline
\end{tabular}

of the cantilever, keeping the tip of the NW down, was introduced. With these two forces a good agreement of both the measured strain $\epsilon_{\perp}$ and the tilt variation $\alpha$ along the NW axis is obtained.

To reproduce the strain measured in NW2, the simulated wire was elongated by $12 \mathrm{~nm}$ through a force $F_{1}$ of $6.30 \mu \mathrm{N}$. The measured strain $\epsilon_{\perp}$ and the diagonal strain component $\epsilon_{y y}$ from the FEM simulation are shown in figure 5(c), again with an in-scale sketch of the simulated geometry. The highest strain in the radial direction, measured in the NW tip, was around $-0.14 \%$. The bending values are presented in figure 5(d). The bending in the simulation was achieved by a force $F_{2}$ of $0.007 \mu \mathrm{N}$, which resulted in a displacement of the NW tip's front face by $12 \mathrm{~nm}$. In comparison to NW1 where the orientation of the front face was kept fixed, for NW2 this surface was allowed to rotate around the $x$-axis. This accounts for the different tilt angles of the NW bottom and tip seen in figure 5(d), while for NW1 the tip and bottom show the same tilt (see figure 5(b)). As can be seen from the simulation results in figure $5(\mathrm{~d})$, the curvature has a maximum after the end of the amorphous shell. This can be understood since the part of the NW which is more supported by the shell, i.e. stiffer due to the bigger diameter, shows indeed only a small bending distribution. Where the support of the tapered shell is fading, higher bending starts to occur.
From the FEM simulations of the measured strain and the measured tilt variations, the complete strain state becomes accessible, and we see some interesting features in the resulting plots in figure 6: the tensile diagonal strain component $\epsilon_{z z}$ is the dominant one, reaching maximum values of $1.16 \%$ and $0.95 \%$ for NW1 and NW2, respectively. Even for relatively small bending the other strain components are nonzero, and the strain state is therefore not purely uniaxial. In particular, due to the bending, finite shear strain components occur, which is shown in figure 6. Furthermore, the strain variation along the wire length depends on the tapering and the tapered amorphous shell. An additional stiffening of the wires happens due to the shell, so that both the Poisson terms $\epsilon_{x x}$ and $\epsilon_{y y}$, as well as the shear terms, vary along the wire. Particular kinks and wide variations occur towards the thin tip of the wire, as well as at the position where the shell ends. This is shown in figure 6: the shaded areas depict the variation of the diagonal strain components and the solid lines of equal colour represent the average values. For the shear terms the variations within their radial distributions are indicated as error bars. Note that these bars do not actually represent an uncertainty, but rather reflect a systematic variation of the values within the wire cross section due to the bending. This can also be seen in the cross section insets displayed for the middle of the NW. 


\section{Comparison to Raman measurements}

Using the full strain tensor obtained from detailed FEM calculations, we are able to simulate the Raman shifts following the approach of Peng and De Wolf [27, 28], and compare the results to the experimental values, as shown in figure 7 for NW1 and NW2 in panels (a) and (b), respectively. The simulated variation of the Raman shift along the axis is depicted as a blue line. A measure of the maximum and minimum values of the simulated Raman is given by the blue areas. The derived strain values reproduce the measured Raman shifts (black diamonds) very well. The data are also compatible with a linear relationship $\Delta \Omega \cong-k \epsilon_{z z}$ between Raman response and the axial strain component $\epsilon_{z z}$ with a linearity factor of $k=434 \frac{1}{\mathrm{~cm}}$ [29], and we can also compare the data in another way. In figures 7 (c) and (d), the strain values $\epsilon_{\|}$along the NW axis obtained using this linear relation from the Raman shifts are plotted against the strain values obtained from the XRD/FEM analysis, again showing a very good match. Table 2 lists the values obtained from the Raman experiment $\Delta \Omega$, as well as strain values $\epsilon_{\|}$calculated using the conversion factor $k$ and those derived from the XRD analysis. Moreover, from the XRD results, we see that the strain state is not purely uniaxial but actually more complex, and we can determine even minor shear components not accessible to Raman analysis for single NWs.

\section{Summary and conclusion}

Prestrained germanium NWs have been investigated using nanofocused $\mathrm{x}$-ray diffraction to provide complementary results for $\mu$-Raman scattering experiments on the very same nanometer-scaled objects. From the position of the recorded

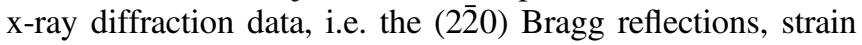
values in the plane perpendicular to the NW growth direction were deduced. This results in the strain distribution along the NW axis with a real-space resolution of approximately $400 \mathrm{~nm}$, taking into account the focused x-ray beam size. With the presented approach a significant strain along the wire growth direction $\epsilon_{\|}$of $1.13 \%$ can be introduced into the NWs, and a strain gradient was found. Although the static strain obtained in our work is not enough to reach direct band gaps in Ge it enables further work, possibly by dynamically straining the NWs. The measured strain values were found by simulating the NWs using FEM models, and comparing the resulting strain perpendicular to the growth direction $\epsilon_{\perp}$ with the measured strain state. Taking into account the measured bending of the nanowires yields additional input for the simulations, partially compensating for the fact that only one Bragg reflection was accessible, due to geometric restrictions. From the simulations, the strain gradient along the NWs was explained in more detail by their tapered shape, which is confirmed by high-resolution SEM images. Additionally, an amorphous oxide shell around the crystalline NW's core was found and taken into account for the simulations, which causes strain variations due to a position-dependent stiffening effect. The XRD/FEM results corroborate the Raman analysis using a linear relationship between uniaxial strain and line shift, but allow us to obtain additional insight into the full strain state of the wires.

\section{Acknowledgments}

This work was supported by the European Commission (EC) (NODE cont. no. 015783, CALIPSO cont. no. 312284, AMON-RA cont. no. 214814), and the FWF Vienna (SFB IROn F2507-N08, P23706-N19). We would like to thank the staff of beamline ID01 at the ESRF in Grenoble for assistance during the nanofocus experiments, in particular Hamid Djazouli for his technical support.

\section{References}

[1] Song Y, Zhou H, Xu Q, Luo J, Yin H, Yan J and Zhong H 2011 J. Electron. Mater. 40 1584-612

[2] Wang J and Lee S 2011 Sensors 11 696-718

[3] Basu P, Das N, Mukhopadhyay B, Sen G and Das M 2009 Opt. Quantum Electron. 41 567-81

[4] Ahn Y and Park J 2007 Appl. Phys. Lett. 91162102

[5] Leem J W, Kim Y P and Yu J S 2012 J. Opt. Soc. Am. B 29 357-62

[6] Kim C J, Lee H S, Cho Y J, Kang K and Jo M H 2010 Nano Lett. 10 2043-8

[7] Cao L, White J S, Park J S, Schuller J A, Clemens B M and Brongersma M L 2009 Nat. Mater. 8 643-7

[8] Chelikowsky J R and Cohen M L 1976 Phys. Rev. B 14 556-82

[9] Lazzouni M E and Sham L J 1993 Appl. Phys. Lett. 63 3253-5

[10] d'Avezac M, Luo J W, Chanier T and Zunger A 2012 Phys. Rev. Lett. 108027401

[11] Liu J, Sun X, Camacho-Aguilera R, Kimerling L C and Michel J 2010 Opt. Lett. 35 679-81

[12] Carroll L, Friedli P, Neuenschwander S, Sigg H, Cecchi S, Isa F, Chrastina D, Isella G, Fedoryshyn Y and Faist J 2012 Phys. Rev. Lett. 109057402

[13] Su S, Cheng B, Xue C, Wang W, Cao Q, Xue H, Hu W, Zhang G, Zuo Y and Wang Q 2011 Opt. Express 19 6400-5

[14] Wirths S et al 2015 Nat. Photonics 9 88-92

[15] Etzelstorfer T et al 2014 J. Synchrotron Radiat. 21 111-8

[16] Zhang F, Crespi V H and Zhang P 2009 Phys. Rev. Lett. 102 156401

[17] Sukhdeo D S, Nam D, Kang J H, Brongersma M L and Saraswat K C 2014 Photonics Res. 2 A8-13

[18] Smith D A, Holmberg V C and Korgel B A 2010 ACS Nano 4 2356-62

[19] Lugstein A, Steinmair M, Steiger A, Kosina H and Bertagnolli E 2010 Nano Lett. $103204-8$

[20] Greil J, Lugstein A, Zeiner C, Strasser G and Bertagnolli E 2012 Nano Lett. 12 6230-4

[21] Süess M J, Geiger R, Minamisawa R A, Schiefler G, Frigerio J, Chrastina D, Isella G, Spolenak R, Faist J and Sigg H 2013 Nat. Photonics 7 466-472

[22] http://code-aster.org/

[23] Mocuta C, Stangl J, Mundboth K, Metzger T H, Bauer G, Vartanyants I A, Schmidbauer M and Boeck T 2008 Phys. Rev. B 77245425

[24] Chahine G A et al 2014 J. Appl. Crystallogr. 47 762-9

[25] http://ioffe.ru/SVA/NSM/Semicond/index.html 
[26] Mathioudakis C and Kelires P 2000 J. Non-Cryst. Solids 266 $161-5$

[27] Peng C Y, Huang C F, Fu Y C, Yang Y H, Lai C Y,

Chang S T and Liu C W 2009 J. Appl. Phys. 105083537
[28] De Wolf I, Maes H and Jones S K 1996 J. Appl. Phys. 79 7148-56

[29] Cerdeira F, Buchenauer C J, Pollak F H and Cardona M 1972 Phys. Rev. B 5 580-93 